\title{
LAS LECTURAS QUE DISFRUTAMOS: PRODUCCIÓN EDITORIAL EN CHILE (2000-2012)
}

María Eugenia Domínguez Saúl 


\section{MARÍA EUGENIA DOMÍNGUEZ SAÚL}

Profesora asistente del Instituto de la Comunicación e Imagen de la Universidad de Chile. Phd. en Comunicación de la Universidad de Montreal. Se ha especializado en el campo de las Industrias Culturales. En ese marco, se interesa por la economía política de la comunicación y la cultura. Su trabajo está centrado en los debates críticos sobre producción, circulación y consumo, la independencia en la industria editorial, las políticas culturales y las condiciones de acceso de la población a los bienes y servicios en este campo. 


\title{
LAS LECTURAS QUE DISFRUTAMOS: PRODUCCIÓN EDITORIAL EN CHILE (2000-2012)
}

\author{
Gino Bailey Bergamin', Felipe Espinosa Parra², Rolando Tiemann Hernández ${ }^{3}$ \\ (Colaboradores)
}

Nuestro punto de arranque fue el malestar frente a la enorme deuda de la academia chilena con el libro y con sus lectores. En efecto, la observación de lo producido hasta hoy, con la excepción de los trabajos de Bernardo Subercaseaux y Grínor Rojo, permite constatar que, pese a la preocupación pública respecto de los índices de lectura, a los debates respecto a las políticas del libro, y a la reemergencia en tiempos democráticos de catálogos, editores y publicaciones, el libro chileno vive en sintonía con la Universidad. Esta investigación, junto a las que otros colegas presentan hoy, bajo el alero del Observatorio del Libro y la Lectura, y cuyos resultados parciales presentamos, busca contribuir y generar preguntas que permitan interrogarnos sobre lo que se escribe y lo que se publica.

Poco sabemos de la situación de la cadena del libro, de los modos de producción y lógicas de funcionamiento de los editores y libreros, especialmente pequeños y medianos. Por lo que responder a estas primeras cuestiones permitirá debatir luego respecto de las políticas públicas, las condiciones en que se escribe y las que se lee; el acceso, el consumo, y los gustos de los públicos. Finalmente, de la circulación y del tipo de conocimiento social del que los libros son portadores.

Es desde este punto de partida que interrogamos la producción de libros en Chile en el nuevo siglo. Para ello, revisamos la información disponible, entre los años 2000 y 2012, en los registros de libros en ISBN4.

1. Sociólogo de la Universidad de Valparaíso y Magister en Geografía y Procesos territoriales de la Universidad de Bolonia. Su área de interés se ha concentrado en la investigación sobre el territorio, las formas sociales de representación geográfica, los modelos espaciales y globales. Se ha especializado en el destino de las ciudades portuarias (waterfronts), así como en la economía cultural del territorio. Además de la investigación, ha participado en programas de intervención cultural y evaluación social con comunidades en ciudades menores de Europa y Chile.

2. Sociólogo del Instituto de Sociología de la Universidad de Valparaíso y candidato a Magíster en Comunicación Social del Instituto de la Comunicación e Imagen de la Universidad de Chile. Se ha interesado en temáticas culturales y urbanas, economía política de la cultura e industrias culturales, sociología de las ciudades y desarrollo urbano. Especializado en metodologías de investigación, comportamiento de masas, y problemas en sociedades contemporáneas. Premiado con la beca Canada-Chile Leadership Exchange Scholarship del Gobierno de Canadá (2013).

3. Sociólogo del Instituto de Sociología de la Universidad de Valparaíso y candidato a Magíster en Comunicación Social del Instituto de la Comunicación e Imagen de la Universidad de Chile. Profesor honorario de la Universidad Viña del Mar. Experiencia en investigaciones en industrias culturales. Se ha interesado en teoría sobre consumo, comunicación, publicidad, industria cultural y sociología económica.

4. El ISBN (International Standard Book Number) es un número de serie que se le asigna a cada libro publicado en el país. Para obtener un número de ISBN, el editor inscribe cada título en una base de datos. De 


\section{CONSIDERACIONES SOBRE LA ESTRATEGIA METODOLÓGICA}

El registro ISBN es una fuente fundamental de información. No obstante, el hecho que la inscripción y clasificación posterior sea realizada a partir de la información proporcionada por las empresas e instituciones, sin mecanismos ulteriores que permitan verificar (entre otros datos, la pertinencia de su clasificación por materia), y que dichas bases de datos consideren, no solo las publicaciones de empresas dedicadas profesionalmente a la edición de libros, sino también aquellas provenientes de sectores empresariales, de ONG's, de instituciones públicas y privadas de distinto carácter, como la autoedición: nos obliga a una construcción del corpus que establezca una tipología de los productores.

Esto es, que diferencie aquellos que corresponden al sector editorial, propiamente tal, de aquellas publicaciones correspondientes a otras instituciones o personas. Y es, particularmente, relevante por cuanto se estima, en el caso chileno, una sobreevaluación de la producción de la industria editorial de alrededor de entre un 20 y un $30 \%$ anual.

Del mismo modo, y con el propósito de diferenciar con claridad un catálogo editorial chileno, excluimos el libro funcional a propósitos educativos y legales (manuales escolares y códigos legales, entre otros).

Ahora bien, observamos los datos extraídos del ISBN bajo dos formas. Así, en la primera parte, analizamos los datos totales de ISBN que se encontraban en informes institucionales para comparar cifras de Chile y América Latina. Posteriormente, en la segunda instancia, realizamos una segmentación de los registros ISBN para enfocarnos en un tipo particular de libros, que creemos, reflejan parte de la industria editorial chilena.

\section{PRODUCCIÓN EDITORIAL EN CHILE: ALTAS TASAS DE CRECIMIENTO, ¿POR QUÉ?}

En Chile, la producción editorial registra un crecimiento sostenido en la última década. Según los datos informados por la Cámara Chilena del Libro5, dan cuenta de un crecimiento del 150\% entre los años 2000 y 2012 (es decir, un promedio de 8\% anual). Allí, los crecimientos más importantes se registran entre los años 2002-2003 y luego entre el 20092011. Los años 2004 y 2006, en tanto, presentan crecimientos negativos de $-8 \%$ y $-1 \%$.

Estas tasas de crecimiento son similares a las que se encuentran en el total de los países de Latinoamérica, lo que indica que el país sigue la tendencia regional. En términos absolutos, el crecimiento significa que hemos pasado de 2.420 libros en el año 2000, a 6.045 para el año 2012.

esta manera, casi la totalidad de los libros publicados tienen este número, que permite identificar en qué país fue escrito, la casa editora, el autor, el año en que fue publicado, y la temática o materia de la que trata el libro. En Chile, desde el 1 de julio de 1993, la Ley No 19.227 sobre Fomento del Libro y la Lectura, impone en su artículo $8^{\circ}$ la obligatoriedad del ISBN a todo libro chileno: Ver: http://www.isbnchile.cl/

5. Institución responsable del registro ISBN en el país. 
Gráfico 1

Libros publicados en Chile por año (2000-2012)

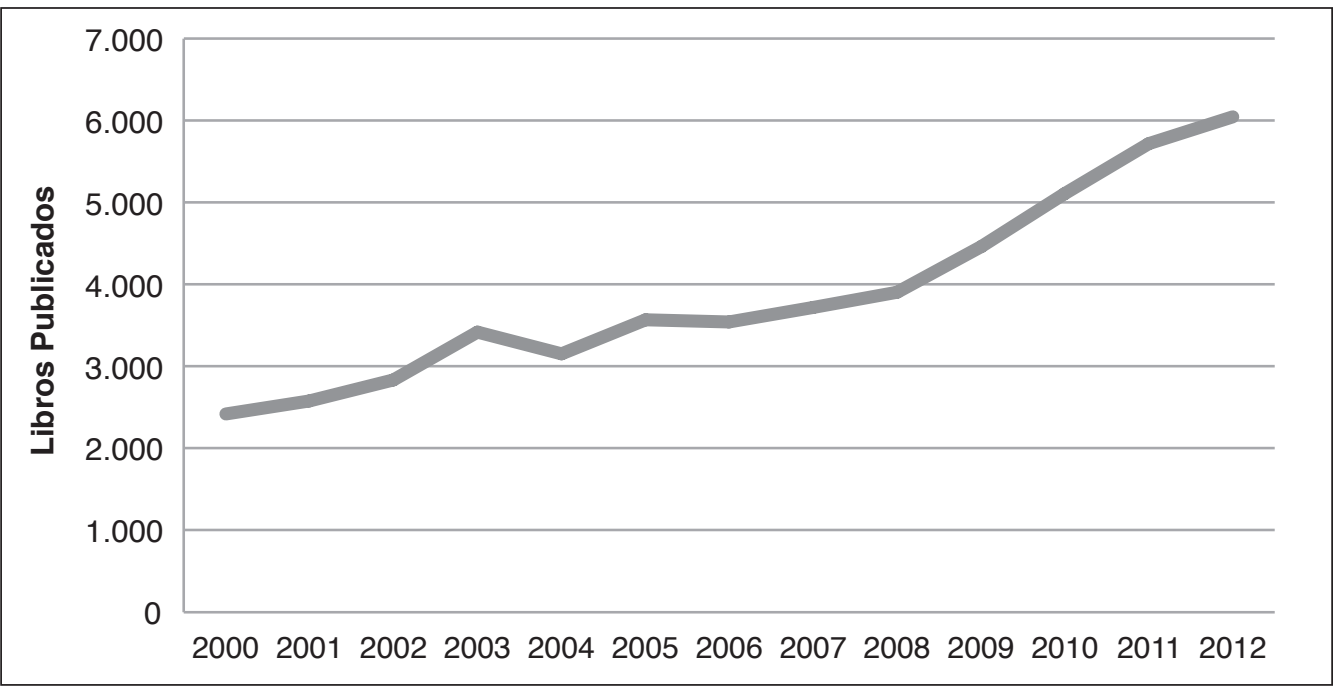

Fuente: Elaboración propia en base a datos de la Cámara Chilena del Libro A.G. (Informe Estadístico 2012 Agencia Chilena ISBN, 2013).

Al observar con mayor detalle las áreas de la producción de libros, considerando la relación entre el número de editores por 100 mil habitantes, se constata la existencia de una industria editorial relativamente constituida y constante. Existen altas tasas de publicación y registro de libros, lo indica el nivel de formalización de la industria. Sin embargo, cerca del 14\% de los libros publicados son en realidad autoediciones.

Las autoediciones son libros publicados por el mismo autor y no a través de una empresa editora, que, por su naturaleza, no tienen asegurada su entrada en el circuito comercial, lo que va a depender en gran medida de las acciones y actividades de difusión que se lleven a cabo. ¿Por qué sucede que una gran cantidad de libros publicados no pasan por un proceso editorial? No es sencillo explicar esto solo a partir de los datos de registro comercial y, por lo tanto, solo se pueden elaborar algunas hipótesis.

La primera es que la cadena de editoriales del país no logra suplir a toda la demanda de autores decididos a publicar. Esto podría estar sucediendo en todo tipo de literatura y en autores para los cuales el oficio de escribir no es su actividad primaria. Además, es importante destacar que si bien el costo de publicación de un libro autoeditado es relativamente bajo, aun así implica al menos el trabajo de impresión, cuyo financiamiento podría provenir de distintas fuentes. Hasta hace un tiempo, el Fondo del Libro del Consejo Nacional de la Cultura y las Artes, que apoya con financiamiento la publicación de obras, no exigía que estas fueran publicadas a través de un agente editorial.

Es probable que una buena cantidad de libros financiados a partir de este mecanismo hayan sido publicados por sus mismos autores. Sin embargo, en los últimos 
años se ha convertido en una exigencia que toda publicación venga de la mano de una editorial, siendo estas las que postulan los libros para ser financiados por este fondo concursable. Esto debería significar que menos libros son autoeditados a partir de este cambio en la regulación, pero los datos indican que la tendencia no ha variado y ha mantenido altas tasas de crecimiento: las autoediciones crecen en total $124 \%$, entre los años 2000-2012, un poco más bajo que el 150\% de crecimiento en el total de las publicaciones.

Las preguntas que surgen, al observar las cifras de libros publicados en Chile, apuntan a conocer las características de esta industria editorial que tiene una posición relevante en América Latina. El fenómeno de los libros autoeditados llama la atención, ya que abarca, como indicamos, el $14 \%$ de las publicaciones, una cifra alta para un país con una industria semiconsolidada en comparación a otros países de América Latina.

\section{1 ¿Sobre qué se escribe?}

En los últimos 13 años se han publicado 50.479 libros en el país. El ISBN asigna una materia, que representa una forma de clasificación temática del contenido de un libro. De acuerdo a los datos, las materias más comunes entre los años 2000-2012, son las de Literatura (31\%) y Educación (16\%). También son relevantes las materias de Tecnología, Arte y Recreación y Derecho, cada una de ellas representan un siete por ciento en estos años. Materias que, en total, representan el 70,2\% del total de los libros publicados en el país.

Al analizar este período, el crecimiento más importante se produce en "Educación", cuyas publicaciones crecen un 305\%, tomando como año base el 2000. Este crecimiento es significativo, ya que pasan de publicarse 350 libros de educación el año 2000, a 1.420 el año 2012. Otro hecho significativo es el crecimiento de los libros de "Literatura", los cuales crecen un $134 \%$.

Los crecimientos por sobre la media, es decir, las materias que crecieron por sobre otras materias, son Lenguas (376\%), Ciencias Filosóficas (265\%), Ciencias Sociales (287\%), Derecho (174\%) y Cs. Aux. Historia (151\%). En estos casos, sobre todo en Ciencias Sociales, el crecimiento es relevante, puesto que pasan de 120 libros el año 2000, a 465 en el año 2012; transformándose en una materia relevante para el total de las publicaciones. El único tipo de libro que decrece en este periodo es el de Religión, el cual pasa de 104 libros publicados en el año 2000, a 89 libros el 2012, existiendo una baja de $-14 \%$.

\subsection{La vida y tiraje del libro chileno}

El número de edición permite analizar la "vida" de los libros, es decir, aquellas obras que perduran en el tiempo y de las cuales se realiza más de una edición. En Chile son pocos, muy pocos, los que subsisten más allá de la primera edición. 
Para analizar esto con mayor precisión, existe otro dato relevante: el tiraje, es decir, la cantidad de ejemplares que se publican con cada edición. Mientras el número de publicaciones nos indica cuántos libros distintos se han inscrito en el registro ISBN, el tiraje nos acerca a la dimensión más cuantitativa, es decir, cuántos ejemplares o libros se publicaron para cada edición. Y al recategorizar estas cifras en cuatro segmentos (de 1 a 1.000 ejemplares; de 1.001 a 3.500 ejemplares; de 3.501 a 5.000 ejemplares; y 5.001 y más ejemplares), podemos observar que rara vez se alcanzan tirajes sobre los 3.500 ejemplares ( $87 \%$ de los tirajes). Sobre ellos, la mayoría promedia los 500 .

Lo anterior significa que el libro puede tener una salida comercial, pero con un tope de ventas. Esta estrategia de pocos era, hasta hace unos años, la forma más económica de producir libros con poco riesgo. Esto podría explicar que, del 87\% de libros con un tiraje de entre 1 y 3.500 ejemplares, el 56\% corresponda a la primera categoría (entre 1 y 1.000 ejemplares). Situación que quiere decir que casi la mitad de los libros publicados en Chile tienen un tiraje que considera una distribución semicomercial.

De acuerdo a distintas informaciones sobre éxitos de venta y best-seller, los libros que alcanzan a llegar a este ranking fluctúan entre los 3.500 y 90.000 ejemplares vendidos. Si esto es cierto, quiere decir que el $87 \%$ de los libros publicados en Chile no tienen posibilidad alguna de llegar a las listas de mejores ventas, por la simple razón de que no tienen el volumen de ejemplares necesarios para abarcar este mercado.

\subsection{Bibliodiversidad ${ }^{6}$ y editores independientes (diverso, pero poco y breve)}

En promedio, el 31\% de los libros tiene un tiraje de 1.001 a 3.500 ejemplares. Sin embargo, la mayoría de los libros que se encuentran en la primera categoría (56\%) realizan un tiraje de menos de 501 ejemplares, lo que confirma la hipótesis de que las editoriales independientes juegan un rol relevante en la conformación de la industria editorial en Chile.

En este caso se ve una relación similar a la que se encontraba en los datos sobre el número de edición de los libros. Existe una gran mayoría de libros publicados, como primera edición, y existe una mayoría de libros con menos de 3.500 ejemplares por edición. Dado el alto grado de formalización de la industria (solo un 14\% son autoediciones), no es arriesgado decir que gran parte de la diversidad de títulos —o bibliodiversidad-, se debe a una red de editoriales más pequeñas con un alto grado de formalización, pero con una limitada capacidad de integración al mercado masivo.

Esto se debe a diversas razones, en su mayoría, a las netamente comerciales, relacionadas con el tamaño de la empresa editorial. Muchos de los editores con los cuales se sostuvieron entrevistas, declararon que su baja capacidad productiva se debe principalmente a las limitaciones financieras; no logrando atraer inversionistas o generar alianzas estratégicas con otras empresas del rubro como distribuidoras o retailers.

6. Es decir, la diversidad de las producciones editoriales puestas a disposición de los lectores. 
Esto puede ser producto también de la falta de información económica sobre el campo, donde salvo algunos artículos o secciones de la prensa, no existe información formal y sistemática que sostenga la idea de la industria editorial como un sector económicamente relevante para el país. Es así como, estas asimetrías de información, sin duda, han perjudicado el crecimiento de una industria que podría estar en mejores condiciones competitivas a nivel latinoamericano. También existen otras razones que explicarían estas cifras, y que tienen que ver con procesos culturales de larga data y que responden a lo que Bernard Miège denomina "lógicas sociales". Estas lógicas dicen relación con formas de hacer y comprender la actividad, que en el caso de la edición de libros responde a la forma en que los editores comprenden su actividad y su rol como agentes culturales.

El libro como objeto cultural genera distintas relaciones que no siempre son predecibles a partir de fórmulas económicas, por ende, se usan estrategias que implican menor riesgo. El libro como objeto o como artefacto, se ha vuelto una de las estrategias de la edición independiente, donde el valor de la obra se da en tanto objeto físico y cultural. Por ello, se prefieren ediciones de bajo tiraje, manufacturadas de manera semiartesanal o semiindustrial, distribuidas a través de redes directas o sin intermediarios, y donde el trabajo de edición, corrección, diagramación y diseño, se limita un número reducido de personas. Esta forma de producción responde en este sentido a lógicas económicas y culturales. Y su manera de comprender la actividad editorial se ve reflejada en las cifras que muestran el total de ejemplares publicados y el tiraje.

\section{LOS EDITORES EN CHILE: MÁS ALLÁ DE LAS CIFRAS DE CRECIMIENTO}

De acuerdo a los datos informados por CERLALC (2010), el número de editores registrados en el año 2009 en Chile llega a 1.032. De acuerdo a nuestros criterios de segmentación, para este año, los agentes editores son solo 163. Esto corresponde solo a un $16 \%$ de los editores registrados en el total de los datos, lo que significa que una alta proporción de los editores corresponden a universidades, autoeditores, agencias gubernamentales y organizaciones no dedicadas exclusivamente a la actividad de edición. Lo que es coherente con los datos de segmentación que indicaban que solo un $24 \%$ de las publicaciones corresponden a libros destinados al mercado de librerías.

Al analizar el total del período 2000-2012, encontramos que son 428 los agentes editoriales registrados bajo los nuevos criterios. Estos datos reflejan la actividad editorial en el país, por lo que 428 corresponde al número total de editoriales que tuvieron actividad durante estos años. Para el año 2000, fueron 77 las editoriales que registraron actividad bajo nuestros criterios de selección. Al año 2012 estas crecieron a 215 , siendo el promedio de este período 134 editoriales, y han registrado un crecimiento importante en el período (179\%), tomando como año base el 2000, lo que se ajusta 
a los crecimientos generales de la industria. Las mayores alzas se producen entre los años 2005-2006, donde se registra un crecimiento de 21\%, y 2011-2012, donde el crecimiento es de un 28\%. Los años 2004 y 2010 representan años de crecimiento negativo con un $-3 \%$ y $-1 \%$, lo que explicaría en parte los altos crecimientos en los años posteriores.

\section{Gráfico 2}

Crecimiento de la actividad editorial (2000-2012)

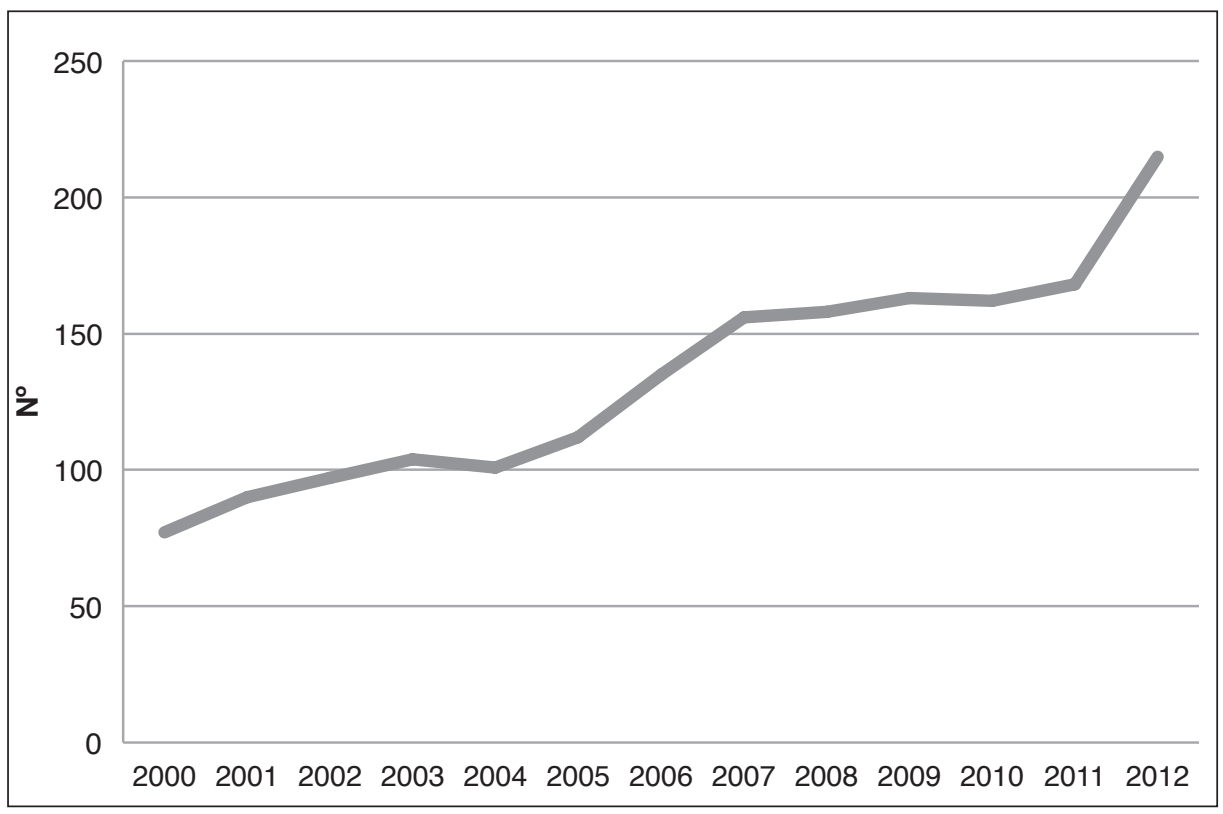

Fuente: Elaboración Propia

El crecimiento de la actividad editorial es consistente con el crecimiento en el número de publicaciones. Mientras en el año 2000 se publicaron 518 títulos y 77 editoriales presentaron actividad, para el año 2012 se publicaron 1.506 títulos y 215 agentes editoriales mostraron actividad.

Salvo algunas variaciones dentro de este período, la proporción se mantiene entre 6 y 8 títulos por editorial. Esta cifra, sin embargo, es hipotética y solo sirve para ilustrar la proporcionalidad del crecimiento en ambos segmentos, ya que como se verá más adelante los libros publicados no se distribuyen de manera uniforme entre las editoriales. El gráfico siguiente muestra el comportamiento del crecimiento en la actividad editorial entre los años 2000-2012 tanto en títulos publicados como en número de editoriales. 
Gráfico 3

Crecimiento de los títulos y editoriales (\%)

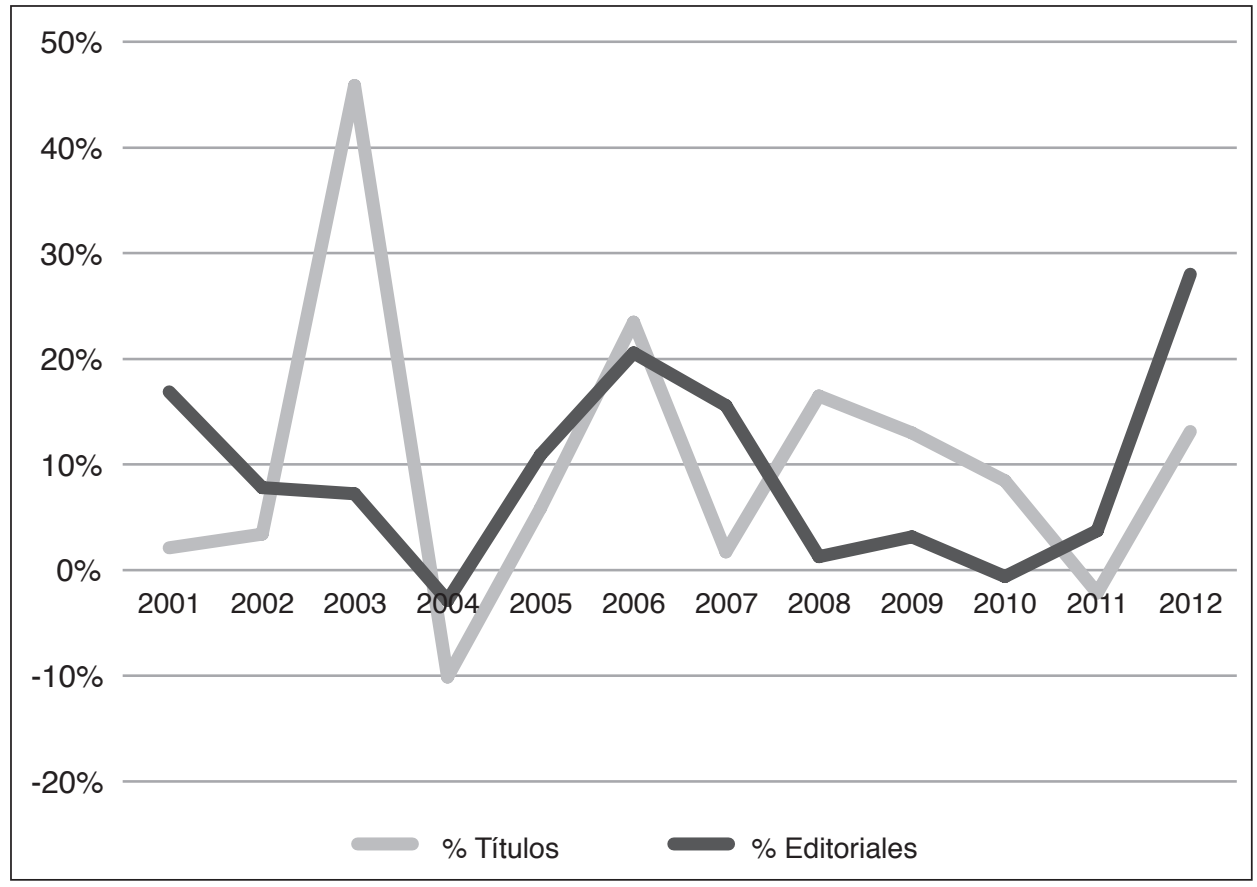

Fuente: Elaboración Propia

Este gráfico refleja el crecimiento de títulos entre los años 2002-2003, mientras que la actividad de las editoriales para este mismo período, baja. Esto significaría una mayor concentración de editoriales en estos años. Sin embargo, para los años 2011-2012 el crecimiento de las editoriales es mayor que el de los títulos.

Observemos enseguida la longevidad y el tamaño de las editoriales. Respecto del primer factor (a partir de la información sobre los años de funcionamiento o actividad que registran los agentes editores en un período de tiempo determinado), constatamos que el 16\% de los agentes editores han tenido solo un año de funcionamiento. El 16\% de ellas son editoriales nuevas que abrieron en los últimos dos años. Esto viene a corroborar las cifras de crecimiento de editoriales vistas en el punto anterior.

Por otro lado, cerca del 70\% de las editoriales de este segmento ha registrado actividad, solo entre uno y cuatro años, lo que indica que gran parte de estas empresas no tienen la capacidad para perdurar en el tiempo. Estas cifras nos indicarían que si bien existe un crecimiento de la actividad editorial, esta no es constante al analizarlo desde las lógicas particulares de cada editorial. Tan solo un 2,8\% de las editoriales de este segmento registra actividades durante todo el período analizado.

Por lo tanto, de las 428 editoriales que registran actividad, solo 127 de ellas lo hacen por más de cuatro años. Esto puede tener varias explicaciones, como el hecho de que algunas editoriales se plantean como proyectos culturales con el fin de levantar un 
catálogo específico. Luego de agotar este catálogo, el proyecto editorial desaparece o se convierte. Este tipo de fenómenos es común en el mundo cultural, como en el cine o la música. Sin embargo, al tratarse de empresas con un giro o personalidad jurídica, sometida a regulaciones como los derechos de autor, esto puede significar un problema. Por lo tanto, la industria editorial que muestra cifras positivas en los datos macro es más compleja de lo que se observa al analizar sus especificidades.

\section{Gráfico 4}

Años de funcionamiento de empresas editoriales en el período 2000-2012

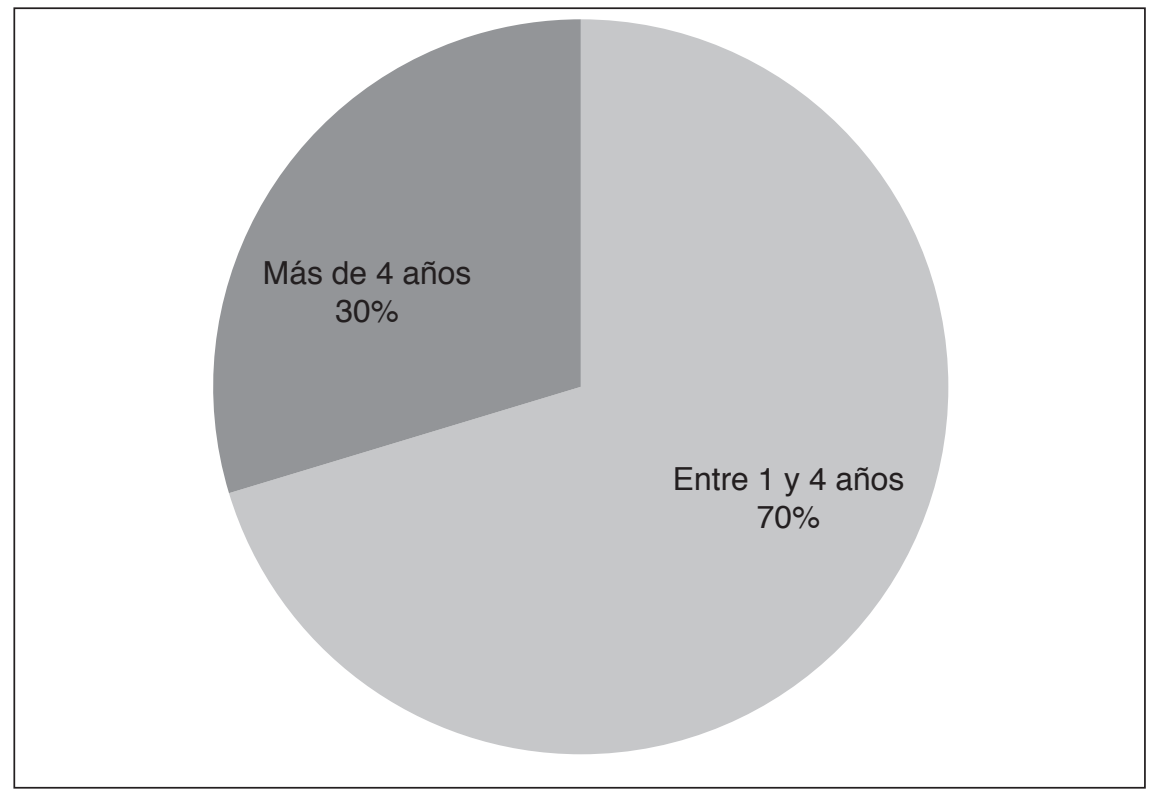

Fuente: Elaboración propia.

Otra explicación, en esta misma línea, se debe a que algunas editoriales han cambiado su nombre en los registros ISBN, sin que esto signifique la desaparición de la empresa. Aunque - salvo excepciones - la limpieza de datos consideró este fenómeno y uniformó los nombres de las editoriales para evitar la "duplicación" de editoriales, la frecuencia más común (moda) de funcionamiento de las editoriales es de dos años y el promedio es de cuatro. Otra forma de presentar estos datos es que el 84\% de las editoriales ha funcionado más de un año, 17\% más de siete años, y un 10\%, más de diez años.

El segundo aspecto, el tamaño de las editoriales, lo observamos a partir del volumen de sus catálogos. Si consideramos la estructura de funcionamiento del mundo de la producción editorial en Chile, al enfocarse en el 10\% de las editoriales con mayor trayectoria o longevidad, nos encontramos que son 43 las editoriales que tienen diez o más años de funcionamiento. En total estas editoriales, las más antiguas, publicaron un $56 \%$. Sin embargo, la antigüedad de la editorial no asegura un catálogo de gran volumen, ya que entre estas editoriales existen algunas cuyo catálogo es tan reducido como 
15 títulos, hasta otras con un catálogo extenso de 915, en el caso de LOM, que es la que más títulos registra.

En esta perspectiva, constatamos que solo el 3\% de las editoriales ha registrado 200 o más títulos durante los años de estudio. La mayor frecuencia de títulos publicados (moda) es de 2 títulos, y el promedio es de 29 títulos publicados en este período de tiempo. El 23\% de las editoriales ha registrado menos de tres títulos en los trece años de estudio. Por otro lado, el $61 \%$ las editoriales que registran actividad lo han hecho con menos de diez títulos, mientras que el 39\% registra más de diez.

Gráfico 5

Títulos registrados por las editoriales 2000-2012

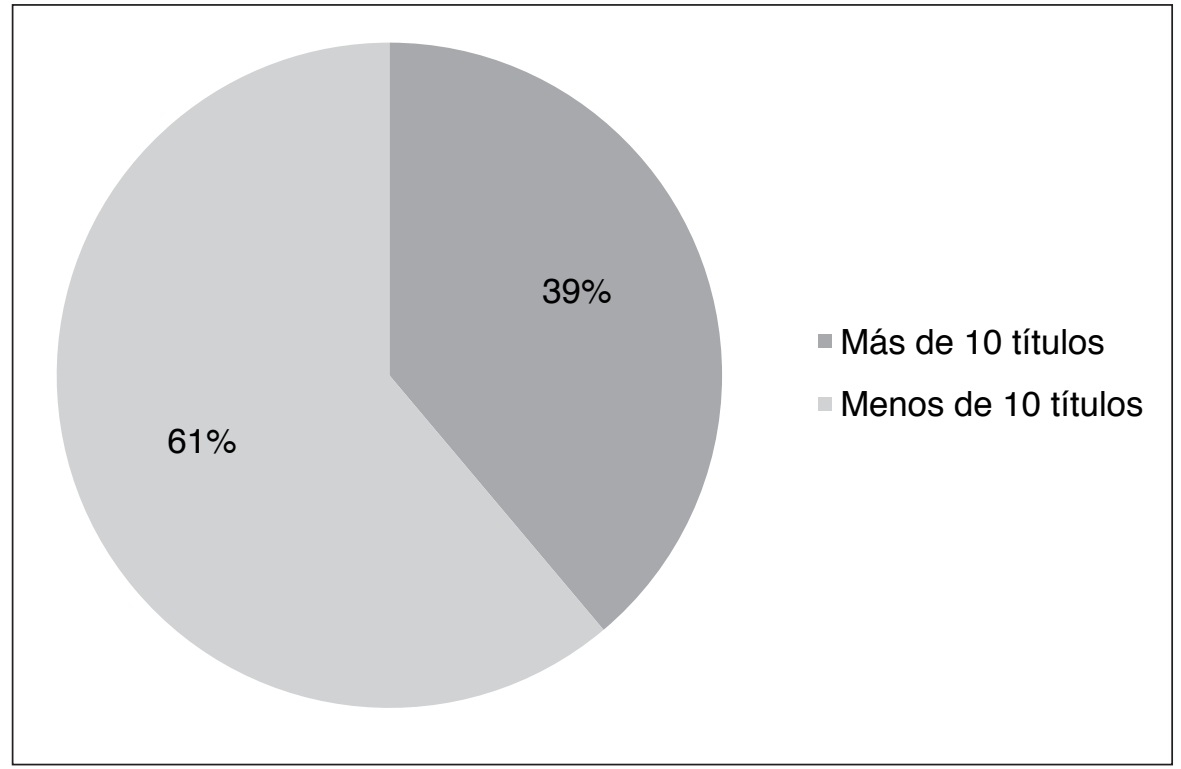

Fuente: Elaboración propia.

Considerando que este estudio toma como período de análisis trece años, el hecho de que más de la mitad de las editoriales solo registran menos de diez títulos parece significativo. Desde este punto de vista, podemos afirmar que las editoriales nacionales tienen una muy baja producción, con menos de un libro anual. Por otro lado, en la punta más alta de esta pirámide, encontramos que un 2,5\% de las editoriales tienen un registro mayor o igual a 200 títulos. Otras diecinueve editoriales, es decir un 4\% del total, registran más de 100 títulos. De acuerdo a lo anterior, en nuestro país existen cuatro tipos de editoriales: una alta proporción que publica menos de 50 libros (89\%), otro segmento que publica entre 50 y 99 libros (4\%), otro que publica entre 100 y 199 libros (4\%), y un último segmento, que publica más de 200 libros (3\%). Las treinta editoriales que se encuentran en la punta de la pirámide conforman el $62 \%$ de la producción editorial chilena. 
En resumen, los datos encontrados nos indican algunos hallazgos interesantes.

- Las cifras en Chile muestran que está bien posicionado en el contexto latinoamericano, con una gran cantidad de editores y títulos publicados por habitante.

- Las publicaciones se concentran en materias específicas como educación, literatura, derecho, entre otras. Además se da cuenta que la mayoría de los libros son primera edición y con un muy bajo tiraje.

- El 70\% de las editoriales funcionan entre uno y cuatro años, y el 61\% tiene un catálogo en este período de menos de diez libros (lo cual es muy bajo en términos de productividad).

- El crecimiento de la industria se explica, en parte, por una gran cantidad de editoriales que nacen anualmente (aunque muchas desaparecen en corto tiempo). Lo anterior explica una alta bibliodiversidad, pero de bajo tiraje.

- Finalmente, en la punta de la pirámide existe una industria editorial consolidada que funciona de manera permanente.

\section{REFLEXIONES FINALES}

El nivel y el número de publicaciones, la diversidad de agentes editoriales, y la diversidad temática existente, permiten sostener que existe un auge de la industria editorial en Chile. Ello implica también que existen nuevos espacios —al menos en potencia— para nuevos públicos interesados en los libros.

Dentro de la diversificación de los temas abordados aparecen las novelas históricas, biografías de figuras públicas del deporte, la política y los medios; novelas del género policial o suspenso, terror y ciencia ficción; libros de ensayos, periodismo de investigación, autoayuda y autobiográficos; así como una amplia gama de libros útiles, manuales, enciclopedias, libros educativos e infantiles. También se han publicado y republicado textos históricos, especialmente respecto de la historia reciente (derechos humanos, movimientos sociales y resistencias a la dictadura) al desarrollo cultural, social y político.

Lo anterior ha permitido que la industria del libro se desarrolle de manera activa, lo que, sumado a nuevas cadenas de distribución, en el retail, librerías y nuevos espacios de venta como ferias de libros, encuentros u otros eventos, han permitido que desde la década de los noventa el mundo editorial viva un aparente auge.

No obstante, existen condiciones que estarían afectando a la industria del libro, entre ellas, la baja cantidad de personas que adquieren libros y lo que probablemente tiene que ver con la baja calidad del alfabetismo en Chile.

Nuevamente sorprende la actualidad de los planteamientos de Domingo Sarmiento para comprender lo que pasaba en la industria editorial hace 150 años. En efecto, Sarmiento señalaba que un libro escrito en Chile tiene "por lectores (potenciales), con pocas excepciones, sólo a los habitantes del país; y aunque estos sean dos millones los libreros, saben que una edición no encuentra colocación entre nosotros (salvo los libros de educación) por más de 500 ejemplares" (Subercaseaux, 200). Sus palabras hablan de la actualidad de la producción editorial. 
Así, de acuerdo a diversos editores consultados, el número de ejemplares por edición no ha cambiado mucho. Es más, un libro editado por un agente independiente rara vez alcanza ventas de más de 200 ejemplares, lo que es significativamente poco, pensando que el país hoy cuenta con más de dieciséis millones de personas.

Una de las condiciones que sí ha cambiado en estas dos últimas décadas analizadas, y que marca una diferencia sustancial con el período de la dictadura, corresponde al "nivel de apertura de la sociedad y del pensamiento". Con el retorno de la democracia se liberó la censura, pero también las trabas económicas que han promovido un nuevo tipo de globalización en la cual el mercado ha sido protagonista.

\section{BIBLIOGRAFÍA}

Canoy, M., Van Ours, J.C. y Van der Ploeg, F., "The Economic of Books", en Ginsburgh, V., Throsby, D., Handbook of the Economics of Art and Culture, Oxford: UK: North-Holland, 2006, 721-759.

Cámara Chilena del Libro AG, Informe Estadístico 2012 Agencia Chilena ISBN, Santiago: Cámara Chilena del Libro A.G., 2013.

CERLALC, El espacio iberoamericano del libro 2010, Santiago, Chile: CERLALC, 2010.

CERLALC (R. Uribe Schroeder, Ed.), El espacio iberoamericano del libro, Madrid: FAS, 2006.

Subercaseaux, B., Historia del Libro en Chile (Alma y Cuerpo), 2ª edición, Santiago, Chile: LOM Ediciones, 2000.

Coedición de la Fundación Chile Veintiuno y la Asociación de Editores de Chile, Una política de Estado para el libro y la lectura, Santiago: Editorial Universitaria, 2005.

Consejo Nacional de la Cultura y las Artes, ANUARIO DE CULTURA Y TIEMPO LIBRE. RECOPILACIÓN DE OCHO AÑOS. 2003 al 2010. Sectores destacados, Departamento de Estudios, Sección de Estadísticas, Santiago: CNCA, 2011.

Domínguez, M. E., Les éditeurs indépendants au Chili (2000-2005). Un développement contemporain des industries culturelles. Thèse présentée à la Faculté des études supérieures en vue de l'obtention du grade de Philosophiae Doctor (Ph.D.) en sciences de la communication, Montréal, Canadá: Université de Montréal, 2010.

Shehu, E., Protska, T., Schmidt-Stöling, C., Clement, M., y Blömeke, E., "The influence of book advertising on sales in the German fiction book market", Journal of Cultural Economics, 2013.

Subercaseaux, B., Historia del Libro en Chile (Alma y Cuerpo), $2^{a}$ edición, Santiago, Chile: LOM Ediciones, 2000. 